\title{
Cambios en el pronóstico a largo plazo de la hipertensión arterial pulmonar
}

\author{
ANDRÉS ENRÍQUEZ ${ }^{1}$, PABLO CASTROํ․ PABLO SEPÚLVEDA², \\ HUGO VERDEJO $^{1}$, DOUGLAS GREIG ${ }^{1}$, LUIGI GABRIELLI ${ }^{1}$, \\ MARCELA FERRADA ${ }^{1}$, CAROLINA LAPOSTOL ${ }^{2}$
}

\section{Changes long term prognosis of 17 patients with pulmonary artery hypertension}

Background: Pulmonary artery hypertension $(P A H)$ is a progressive disease with high mortality. Major advances had been made in the treatment of this condition during the last decade. Aim: To characterize the clinical evolution and mortality of a cohort of Chilean patients. Material and Methods: Seventeen patients with PAH diagnosed in the last 10 years in two Chilean hospitals were enrolled. Measurements at diagnosis included hemodynamic variables and 6-minute walk test. The patients were followed clinically for 3 years and the observed mortality was compared with that predicted by the prognostic equation proposed by the historic registry of the National Institutes of Health (NIH). Results: The mean age of patients was 45 years and $80 \%$ had an idiopathic PAH. The mean median pulmonary artery pressure was $57 \pm 15$ $\mathrm{mmHg}$, the cardiac index was $2.4 \pm 0.7 \mathrm{l} / \mathrm{min} / \mathrm{m}^{2}$ and the right atrial pressure was $12 \pm 8 \mathrm{mmHg}$. The 6-minute walk distance was $348 \pm 98 \mathrm{~m}$. All patients received anticoagulants. Eighty two percent received ambrisentan, $12 \%$ received bosentan, $29 \%$ received iloprost and $24 \%$ sildenafil. At the end of follow-up only 3 patients had died, with an observed survival rate of 88,82 and $82 \%$ at 1,2 and 3 years, respectively. In contrast, the survival calculated according to the predictive formula of the NIH was 67, 56 and 45\%, respectively. Among surviving patients, an improvement in exercise capacity was observed after one year $(p<0.05)$. Conclusions: The observed survival rate was significantly better than that estimated according to historical data. Furthermore, therapy was associated with an improvement in functional capacity after one year. This prognostic improvement is consistent with data of other contemporary registries published after the NIH Registry.

(Rev Med Chile 2011; 139: 327-333).

Key words: Drug therapy; Hypertension, pulmonary; Survival rate.

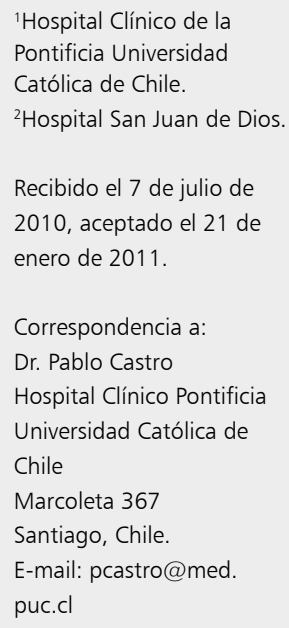

L a hipertensión arterial pulmonar (HAP) es una enfermedad progresiva con una alta mortalidad. El tratamiento de esta condición ha evolucionado considerablemente en años recientes. En la última década un total de 6 drogas específicas para el tratamiento de la HAP idiopática han recibido aprobación por la Food and Drugs Administration (FDA) de los Estados Unidos de Norteamérica, comenzando con el epoprostenol en $1998^{1}$, al cual se han agregado en forma subsi- guiente una prostaciclina subcutánea ${ }^{2}$, una prostaciclina inhalada ${ }^{3}$ y 2 clases de fármacos orales; los antagonistas de los receptores de endotelina ${ }^{4-6}$ y los inhibidores de la fosfodiesterasa tipo $3^{7}$.

La información relativa a la historia natural de la HAP deriva de un registro nacional conducido en Estados Unidos de Norteamérica a comienzos de la década 1980-89 por el US National Institutes of Health (NIH). En dicho registro, 187 pacientes con hipertensión pulmonar primaria (HAP idio- 
pática en la clasificación actual) fueron enrolados en 32 centros clínicos fueron caracterizados y seguidos por un período de hasta 5 años ${ }^{8}$. Este estudio confirmó que el pronóstico de la enfermedad es pobre, con una sobrevida media de 2,8 años después del diagnóstico y tasas de sobrevida a 1,3 y 5 años de 68,48 y $34 \%$ (Figura 1). Series de Japón, India y México sugieren resultados similares, con una sobrevida media en el rango de 2 a 3 años.

El estudio del NIH mostró además que la mortalidad en pacientes con HAP idiopática está estrechamente asociada a tres variables hemodinámicas que reflejan la repercusión sobre la circulación derecha: presión de arteria pulmonar (PAP), presión media de aurícula derecha e índice cardiaco (IC) (Figura 2). A partir de estas variables se dedujo una ecuación pronóstica que permite estimar la sobrevida en un paciente determinado y la cual ha sido validada prospectivamente por estudios posteriores?.

El presente estudio descriptivo tiene como objetivo caracterizar la evolución clínica y mortalidad de los pacientes con HAP con las herramientas terapéuticas actuales y comparar la sobrevida observada con la sobrevida estimada a partir de la serie histórica del NIH.

\section{Métodos}

Se incluyó pacientes portadores de HAP (idiopática, asociada a enfermedad del tejido conectivo, asociada a VIH) diagnosticados entre 1999 y 2005 en los hospitales San Juan de Dios y Hospital Clínico de la Pontificia Universidad Católica de Chile.

HAP fue definida como una PAP media $>25$ $\mathrm{mmHg}$ en reposo y una $\mathrm{PCP}<15 \mathrm{mmHg}$ obtenidas por cateterismo cardiaco derecho. Se efectuó test de vasorreactividad en la mayoría de los casos y se definió como respuesta vasodilatadora positiva a una reducción de la PAP media $>10 \mathrm{mmHg}$ a menos de $40 \mathrm{mmHg}$, con aumento o sin variación del IC.

La HAP fue clasificada en acuerdo con la actual clasificación en idiopática, familiar, asociada a enfermedad tejido conectivo (ETC) o asociada a infección por VIH. La fecha del diagnóstico fue considerada como la fecha del cateterismo cardiaco confirmatorio.

La evaluación inicial incluyó medición de variables hemodinámicas y test de caminata de 6 minutos, además de los datos demográficos y clíni- cos. También se realizaron exámenes destinados a descartar otras causas de hipertensión pulmonar: Radiografía de tórax, angioTAC pulmonar y/o cintigrama pulmonar $\mathrm{V} / \mathrm{Q}$, pruebas de función pulmonar, serología reumatológica, pruebas hepáticas y serología de VIH.

Los pacientes fueron seguidos clínicamente a intervalos de 3 meses por un total de 3 años.

La sobrevida observada fue comparada con la sobrevida a 1,2 y 3 años predicha según la fórmula del registro histórico del $\mathrm{NIH}^{8}$ :

$$
\mathrm{A}(\mathrm{x}, \mathrm{y}, \mathrm{z})=\mathrm{e}^{(0.007325 \mathrm{x})+(0.0526 \mathrm{y})-(0.3275 \mathrm{z})}
$$

donde $x$ es la PAP media, y es la presión media de la $\mathrm{AD}$ y $\mathrm{z}$ es el índice cardiaco. La probabilidad de sobrevida a 1,2 y 3 años está dada por lo siguiente:

$$
\begin{aligned}
& \mathrm{P}(1)=0,75^{\mathrm{A}} \\
& \mathrm{P}(2)=0,65^{\mathrm{A}} \\
& \mathrm{P}(3)=0,55^{\mathrm{A}}
\end{aligned}
$$

Las variables cuantitativas fueron descritas usando media y desviación estándar y las variables categóricas en forma de porcentajes. Para evaluar los cambios de sobrevida respecto a la cohorte histórica se empleó una modificación de $\chi^{2}$ para proporciones. Se consideró significativo un $\mathrm{p}<$ 0,05 (2 colas). Para la comparación de variables continuas se empleó $t$ de Student.

\section{Resultados}

El promedio de edad de los pacientes fue de 45 años, con predominio del sexo femenino (60\%). Desde el punto de vista etiológico, $80 \%$ correspondía a HAP idiopática, seguido de HAP asociada a mesenquimopatía (13\%) y un caso de HAP asociada a VIH (7\%) (Figura 3). Al momento del diagnóstico $60 \%$ de los pacientes estaba en $\mathrm{CF}$ I/II y $40 \%$ en CF III/IV.

Un resumen de las características basales de los 17 pacientes incluidos se muestra en la Tabla 1 .

La PAP media fue de $57 \pm 15 \mathrm{mmHg}$, casi 3 veces el valor normal. El IC fue $2,4 \pm 0,7 \mathrm{l} / \mathrm{min} / \mathrm{m}^{2}$ y la PAD $12 \pm 8 \mathrm{mmHg}$. La distancia caminada en 6 minutos fue en promedio de $348 \pm 98 \mathrm{~m}$. Todos los pacientes recibieron tratamiento anticoagulante. Además, $82 \%$ de los pacientes recibió ambrisentán, $12 \%$ recibió bosentán (ambos bloqueadores del receptor de la endotelina), 27\% recibió iloprost inhalatorio y $20 \%$ sildenafil, $41 \%$ usó furosemida y/o espironolactona. Por último, un tercio de los 

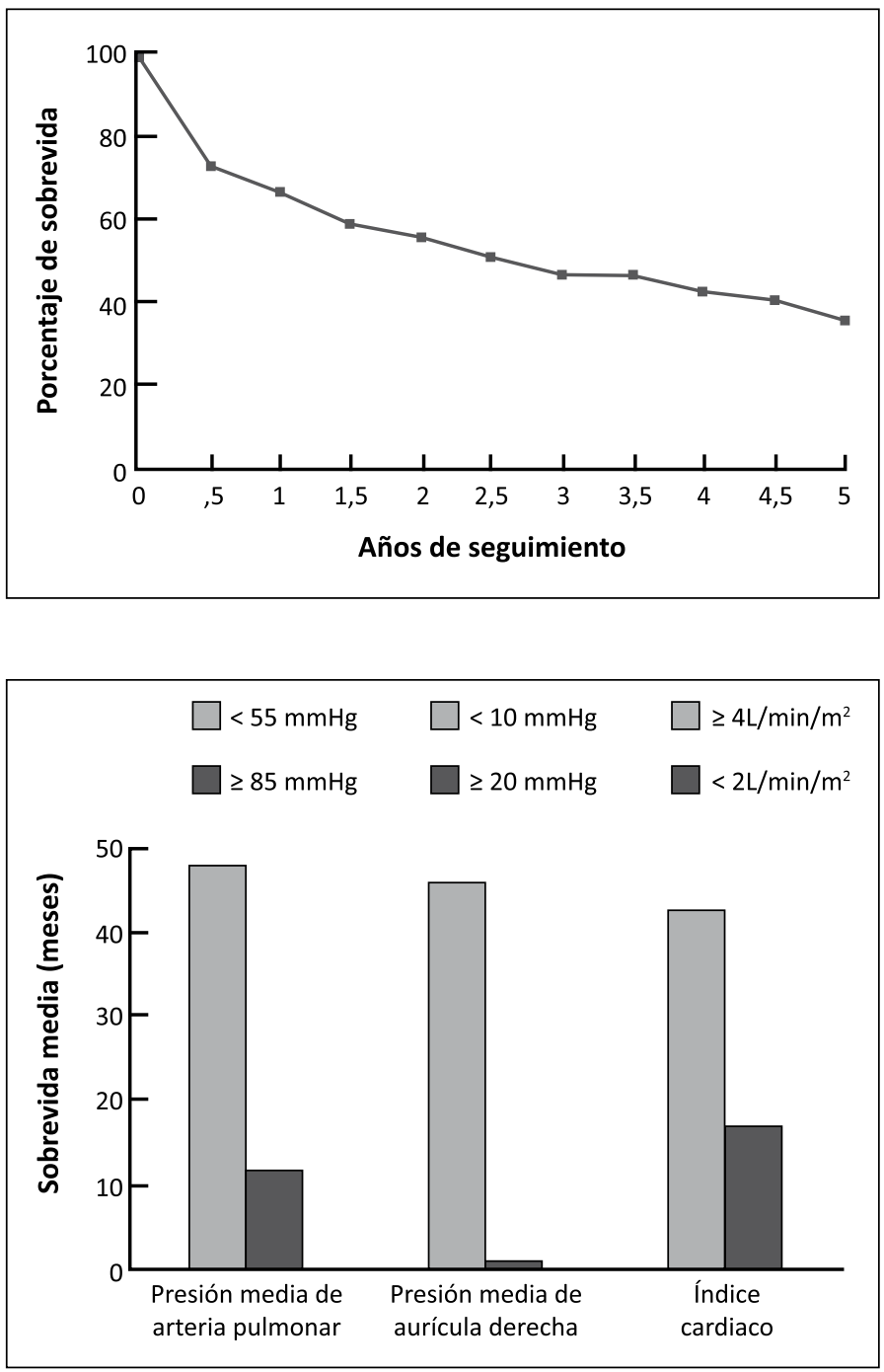

Figura 1. Sobrevida estimada de los pacientes a lo largo del tiempo a partir del cateterismo diagnóstico. Sobrevida media estimada es de 2,8 años. Sobrevida a 1, 3 y 5 años es de 68, 48 y $34 \%$ respectivamente. Reproducido desde (8) con permiso del autor.

Figura 2. Sobrevida media en meses en pacientes con hipertensión pulmonar primaria según 3 variables hemodinámicas. Reproducido desde (8) con permiso del autor.

pacientes recibió terapia combinada con 2 o más drogas específicas (Tabla 2).

Al cabo del período de seguimiento sólo 3 pacientes fallecieron (18\%), arrojando una tasa de sobrevida a 1,2 y 3 años de 88,82 y $82 \%$ respectivamente (Figura 4). En contraste, la sobrevida predicha fue de $67(\mathrm{p}=0,1), 56(\mathrm{p}=0,06)$ y $45 \%$ $(\mathrm{p}=0,006)$.

En los sobrevivientes se observó además una mejoría de la capacidad de ejercicio, con aumento de la distancia recorrida en 6 min desde $348 \mathrm{~m}$ al momento del diagnóstico, hasta $419 \mathrm{~m}$ al cabo de 1 año, cambio que fue significativo $(\mathrm{p}<0,05)$ (Figura 5).

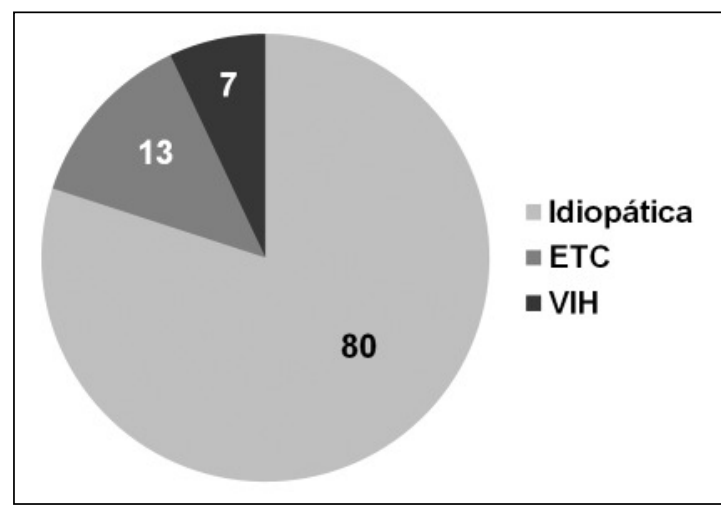

Figura 3. Etiología de la hipertensión pulmonar. ETC: Enfermedades del tejido conectivo. 
Tabla 1. Características basales de los pacientes

\begin{tabular}{|lc|}
\hline & $\begin{array}{c}\text { Pacientes en } \\
\text { estudio }\end{array}$ \\
Sujetos ( $\mathrm{n}$ ) & 17 \\
Relación hombre:mujer & $1: 1,8$ \\
Edad (años) & 45 \\
Clase funcional (\%) & \\
I/II & 53 \\
III/IV & 47 \\
Características hemodinámicas & $57 \pm 15$ \\
PAPm (mmHg) & $12 \pm 8$ \\
PAD (mmHg) & $2,4 \pm 0,7$ \\
IC (L/min*m2) & $348+98$ \\
Test de caminata 6 min (m) & \\
\hline
\end{tabular}

PAPm: presión media de arteria pulmonar; PAD: presión de la aurícula derecha; IC: índice cardiaco.
Tabla 2. Terapias recibidas durante el período de estudio

\begin{tabular}{|lc|}
\hline Terapia & \% \\
\hline Anticoagulación & 100 \\
\hline Calcio antagonistas & 6 \\
Terapias específicas & \\
Ambrisentán & 82 \\
Bosentán & 12 \\
Iloprost & 29 \\
Sildenafil & 24 \\
Terapia combinada & 35 \\
\hline Diuréticos & 41 \\
\hline
\end{tabular}
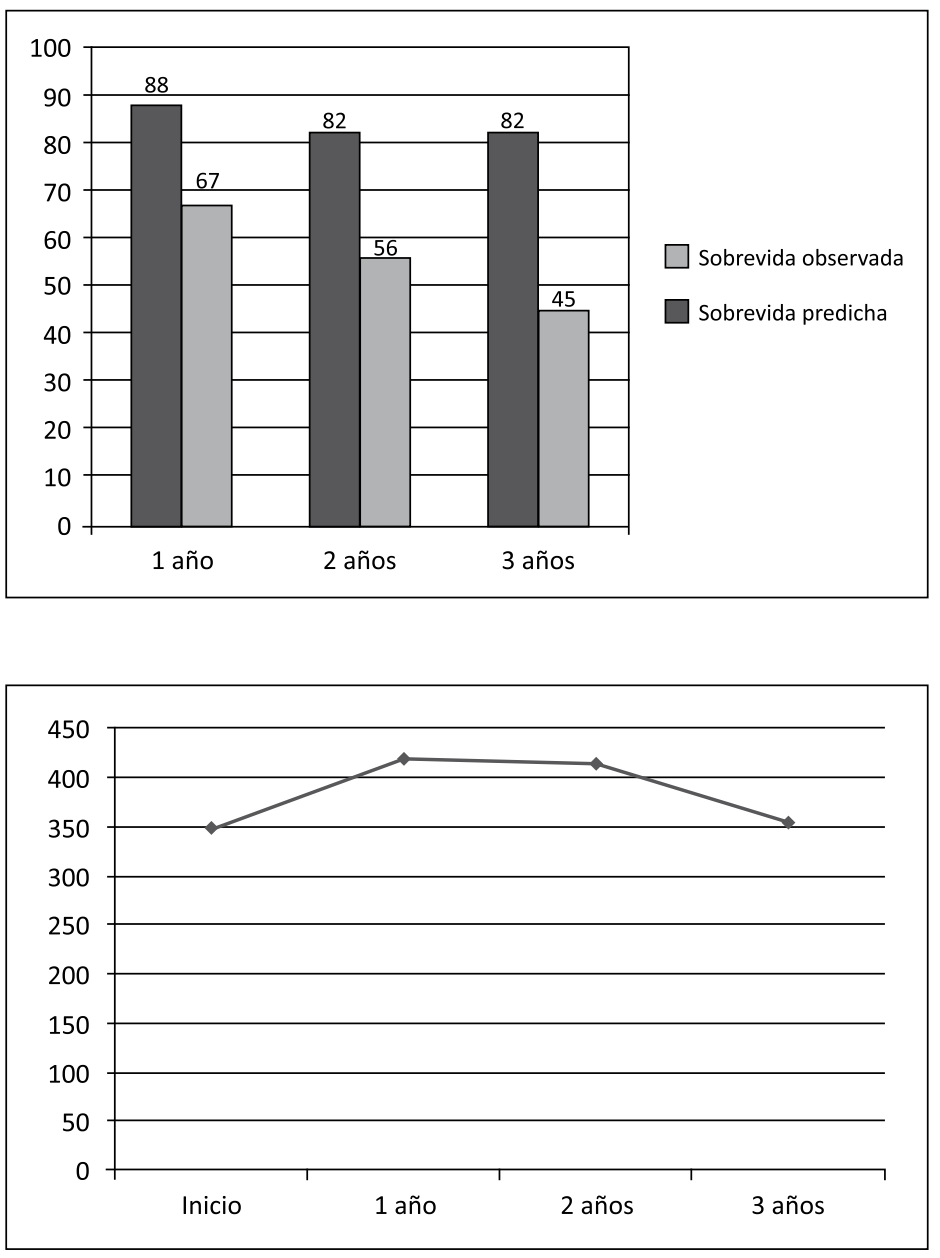

Figura 4. Sobrevida estimada versus predicha.

Figura 5. Cambio en la distancia recorrida en 6 minutos al inicio del estudio, al año y a los 2 y 3 años. 
La tasa de hospitalización durante el período fue de $40 \%$, siendo los motivos principales la insuficiencia cardiaca derecha seguida de síncope.

De los tres pacientes fallecidos, la causa fue falla cardiaca en 2 casos y muerte súbita en el tercero. Los 2 primeros se encontraban en clase funcional III al ingreso y tenían una distancia recorrida en 6 minutos marcadamente reducida (165 y 243 $\mathrm{m})$. Estos pacientes tenían además parámetros hemodinámicos de mal pronóstico, con una sobrevida estimada 1 año por fórmula de $69 \%$ y $67 \%$ respectivamente. El tercer paciente, en cambio, se encontraba en clase funcional II al ingreso, su distancia caminada en 6 minutos estaba sobre el promedio del grupo, $403 \mathrm{~m}$ y sus parámetros hemodinámicos eran de mejor pronóstico, con una sobrevida estimada de $84 \%$ a 1 año.

\section{Discusión}

La sobrevida a 3 años observada en esta serie fue significativamente mayor a la estimada según los datos históricos. Además la terapia se asoció a una mejoría de la capacidad de ejercicio, reflejada por un aumento en la distancia caminada en 6 minutos, hecho que fue significativo a los $12 \mathrm{me}-$ ses. Esta mejoría en el pronóstico a largo plazo de la enfermedad también se observa en otras series contemporáneas (Tabla 3). El registro prospectivo nacional conducido por el French Network of Pulmonary Artery Hypertension incluyó 121 pacientes diagnosticados entre octubre de 2002 y octubre de 2003, constatando una sobrevida a 1 año de $88 \%{ }^{10}$. Por otro lado, el Pulmonary Hypertension Connection Registry, que evaluó 82 pacientes con hipertensión pulmonar grupo I diagnosticados entre 2004 y 2006 en 3 hospitales universitarios de Estados Unidos de Norteamérica, mostró una sobrevida similar, de $85 \%$ a 1 año de seguimiento ${ }^{11}$.

Es posible postular que esta mejoría en la sobrevida estaría explicada en gran parte por la incorporación de las terapias avanzadas desarrolladas y aprobadas con posterioridad a la fecha del registro NIH en la década 1980-89, como posiblemente también por el uso casi universal de anticoagulación en la mayoría de las series contemporáneas.

Existe evidencia en la literatura del impacto favorable de las terapias médicas en la sobrevida de los pacientes con hipertensión pulmonar. El efecto beneficioso del epoprostenol intravenoso ha sido documentado por un trabajo randomizado controlado $^{1}$ y 2 estudios no controlados ${ }^{12,13}$. Estudios observacionales de largo plazo con bosentán ${ }^{14} y$ trepostinil (derivado para uso subcutáneo) también han sugerido una mejoría de la sobrevida en hipertensión pulmonar ${ }^{15}$, aunque no se dispone de estudios randomizados. Por último, estudios openlabel no controlados han demostrado un beneficio

Tabla 3. Comparación con otras series contemporáneas

\begin{tabular}{|lccc|}
\hline & Pacientes & $\begin{array}{c}\text { PH Conection } \\
\text { Registry (USA) }\end{array}$ & $\begin{array}{c}\text { French Network } \\
\text { of PAH }\end{array}$ \\
Fecha & $1999-2005$ & $2004-2006$ & $2002-2003$ \\
Sujetos (n) & 17 & 82 & 121 \\
Edad (años) & 45 & 51 & 53 \\
Mujeres (\%) & 65 & 76 & 57 \\
CF III/IV (\%) & 47 & 83 & 81 \\
Características h-d & & & $49+14$ \\
PAPm (mmHg) & $57+15$ & $51+12$ & $8+5$ \\
PAD (mmHg) & $12+8$ & $10+7$ & $2,4+0,9$ \\
IC (L/min*m²) & $2,4+0,7$ & $2,4-0,8$ & 88 \\
Sobrevida & 88 & 85 & - \\
1 años & 82 & - & \\
3 años & & & \\
\hline
\end{tabular}

CF: clase funcional; h-d: hemodinámicas; PAPm: presión media de arteria pulmonar; PAD: presión de aurícula derecha; IC: índice cardiaco. 
sobre la mortalidad de la terapia anticoagulante con warfarina y el uso de antagonistas del calcio mejora la sobrevida en un pequeño grupo de pacientes que muestran marcada vasorreactividad.

Las drogas usadas en este grupo de pacientes incluyeron ambrisentán, bosentán, iloprost y sildenafil, todas las cuales han demostrado eficacia en estudios controlados sobre el status sintomático, la capacidad de ejercicio y la hemodinamia. El ambrisentán, la droga más usada en esta serie, es un antagonista selectivo del receptor A de endotelina $y$ ha sido evaluado en 2 estudios randomizados (ARIES 1 y 2$)^{6}$, que mostraron beneficio sobre los síntomas, la capacidad de ejercicio, hemodinamia y tiempo hasta el deterioro clínico en pacientes con HAP idiopática y HAP asociada con ETC e infección por VIH. La continuación open-label del estudio mostró durabilidad del beneficio por hasta 1 año desde el inicio de la terapia y fue aprobado para el tratamiento de los pacientes en CF II y III.

Al comparar las características de nuestros pacientes con aquellos de la serie del $\mathrm{NIH}^{8}$ podemos ver que los pacientes en nuestro estudio fueron mayor de edad ( 45 vs 36,4 años). Se confirma el predominio femenino, aunque la relación mujer:hombre fue discretamente menor $(1,5: 1$ vs $1,7: 1)$. La PAP media y el IC fueron similares (57 vs $60 \mathrm{mmHg}$ en el registro NIH y 2,4 vs 2,3 en el registro $\mathrm{NIH}$ ), pero la presión de $\mathrm{AD}$ fue mayor en nuestro grupo ( $12 \mathrm{vs} 9 \mathrm{mmHg}$ ). La proporción de pacientes con síntomas severos (CF III/IV) fue mayor en la cohorte del NIH (71\% pacientes con síntomas III-IV vs $40 \%$ en nuestra serie). La ecuación pronóstica también ha sido validada en grupos de pacientes con menor severidad clínica como la serie de Sandoval et $\mathrm{al}^{9}$, de 61 pacientes. En ésta las características hemodinámicas incluían PAPm 65,5 mmHg, PAD 7,6 mmHg e IC $3,15 \mathrm{~L} / \mathrm{min}^{\star} \mathrm{m}^{2}$ y sólo $39,6 \%$ de los individuos se encontraba en CF III o IV. Además, los pacientes eran más jóvenes (edad promedio 22,6 años) y se excluyó a pacientes con mesenquimopatías, que tienen un pronóstico menos favorable ${ }^{16}$.

La principal limitación de este y otros estudios similares es el uso de un grupo control histórico en lugar de un grupo placebo con el fin de evaluar el impacto real de las terapias médicas en la sobrevida y estatus funcional. Está claro que un diseño semejante sería impracticable por razones éticas dada la naturaleza rápidamente progresiva de la enfermedad en ausencia de intervención. Es interesante notar que, a diferencia de lo observado aquí, la mayoría de los estudios a corto plazo muestran que la distancia caminada en 6 minutos, en ausencia de terapia, muestra una tendencia sostenida hacia el deterioro.

En suma, si bien el pronóstico de los pacientes con hipertensión pulmonar primaria sigue siendo pobre, significativos avances en la fisiopatología y tratamiento de esta condición primaria se han producido en los últimos 10-15 años, con el desarrollo de múltiples terapias efectivas. Los pacientes tienen una mayor sobrevida y una mejor calidad de vida, y muchos de ellos son tratados ahora con fármacos orales.

\section{Referencias}

1. Barst RJ, Rubin LJ, Long WA, McGoon MD, Rich S, Badesch DB, et al. A comparison of continuous intravenous epoprostenol (prostacyclin) with conventional therapy for primary pulmonary hypertension. $\mathrm{N}$ Engl J Med 1996; 334: 296-301.

2. Simonneau G, Barst RJ, Galie N, Naeije R, Rich S, Bourge RC, et al. Continuous subcutaneous infusion of treprostinil, a prostacyclin analogue, in patients with pulmonary arterial hypertension. A double-blind, randomized, placebo-controlled trial. Am J Respir Crit Care Med 2002; 165: 800-4.

3. Olschewski H, Simonneau G, Galie N, Higenbottam T, Naeije R, Rubin LJ, et al. Inhaled iloprost in severe pulmonary hypertension. N Engl J Med 2002; 347: 322-9.

4. Channick RN, Simonneau G, Sitbon O, Robbins IM, Frost A, Tapson VF, et al. Effects of the dual endothelinreceptor antagonist bosentan in patients with pulmonary hypertension: a randomised placebo-controlled study. Lancet 2001; 358 (9288): 1119-23.

5. Barst RJ, Langleben D, Frost A, Horn EM, Oudiz R, Shapiro $S$, et al. Sitaxsentan therapy for pulmonary arterial hypertension. Am J Respir Crit Care Med 2004; 169: 441-7.

6. Galie N, Olschewski H, Oudiz RJ, Torres F, Frost A, Ghofrani HA, et al. Ambrisentan for the treatment of pulmonary arterial hypertension. Results of the ambrisentan in pulmonary arterial hypertension, randomized, double-blind, placebo-controlled, multicenter, efficacy (ARIES) study 1 and 2. Circulation 2008; 117: 3010-9.

7. Galie N, Ghofrani HA, Torbicki A, Barst RJ, Rubin LJ, Badesch D, et al. Sildenafil citrate therapy for pulmonary arterial hypertension. N Engl J Med 2005; 353: 2148-57.

8. D’Alonzo GE, Barst RJ, Ayres SM, Bergofsky EH, Brundage BH, Detre KM, et al. Survival in patients with pri- 
mary pulmonary hypertension: Results from a National Prospective Registry. Ann Intern Med 1991; 115: 343-9.

9. Sandoval J, Bauerle O, Palomar A, Gómez A, MartínezGuerra ML, Beltrán M, et al. Survival in Primary Pulmonary Hypertension: Validation of a prognostic equation. Circulation 1994; 89: 1733-44.

10. Humbert M, Sitbon O, Chaouat A, Bertocchi M, Habib G, Gressin V, et al. Pulmonary Arterial Hypertension in France: Results from a National Registry. Am J Respir Crit Care Med 2006; 173: 1023-30.

11. Thenappan T, Shah S, Rich S, Gomberg-Maitland M. A USA-based registry for pulmonary arterial hypertension: 1982-2006. Eur Respir J 2007; 30: 1103-10.

12. McLaughlin VV, Shillington A, Rich S. Survival in primary pulmonary hypertension: the impact of epoprostenol therapy. Circulation 2002; 106: 1477-82.

13. Sitbon O, Humbert M, Nunes H, Parent F, García G,
Hervé $\mathrm{P}$, et al. Long-term intravenous epoprostenol infusion in primary pulmonary hypertension: prognostic factors and survival. J Am Coll Cardiol 2002; 40: 780-8.

14. McLaughlin VV, Sitbon O, Badesch DB, Barst RJ, Black $\mathrm{C}$, Galiè N, et al. Survival with first-line bosentan in patients with primary pulmonary hypertension. Eur Respir J 2005; 25: 244-9.

15. Tapson VF, Gomberg-Maitland M, McLaughlin VV, Benza RL, Widlitz AC, Krichman A, et al. Safety and efficacy of IV treprostinil for pulmonary arterial hypertension: a prospective, multicenter, open-label, 12-Week trial. Chest 2006; 129: 683-8.

16. Ruiz-Cano MJ, Escribano P, Alonso R, Delgado J, Carreira P, Velázquez T, et al. Comparison of baseline characteristics and survival between patients with idiopathic and connective tissue disease-related pulmonary artery hypertension. J Heart Lung Transplant 2009; 28: 621-7. 